\title{
Momentum transfer resolved memory in a magnetic system with perpendicular anisotropy
}

Keoki A. Seu, ${ }^{1,2}$ S. Roy, ${ }^{1}$ R. Su, ${ }^{1,2}$ D. H. Parks, ${ }^{1,2}$ E. Shipton, ${ }^{3}$ E. E. Fullerton, ${ }^{4}$ and S.

D. Kevan ${ }^{2}$

1) Advanced Light Source, Lawrence Berkeley National Laboratory, Berkeley, California 94720, USA

2) Department of Physics, University of Oregon, Eugene, Oregon 97403, USA

3) Department of Physics, University of California, San Diego, California 92093, $U S A$

4) Electrical and Computer Engineering, University of California, San Diego, California 92093, USA

(Dated: Submitted January 28, 2011)

We have used resonant, coherent soft x-ray scattering to measure wave vector resolved magnetic domain memory in $\mathrm{Co} / \mathrm{Pd}$ multilayers. The technique uses angular cross correlation functions and can be applied to any system with circular annuli of constant values of scattering wave vector $\mathbf{q}$. In our $\mathrm{Co} / \mathrm{Pd}$ film, the memory exhibits a maximum at $\mathbf{q}=0.0384 \mathrm{~nm}^{-1}$ near initial reversal that decreases in magnitude as the magnetization is further reversed. The peak is attributed to bubble domains that nucleate reproducibly near initial reversal and which grow into a labyrinth domain structure that is not reproduced from one magnetization cycle to the next. 
The crossover mechanism of various statistical parameters of a complex system as it evolves from microscopic to mesoscopic length scale is a fascinating topic of research. Examples of such are ubiquitous in nature and are exhibited in diverse soft, hard and biological material systems. In particular, in a magnetic system that exhibits field driven hysteresis, disorder is a key statistical parameter that determines the degree of repeatability of the microscopic domain pattern. Magnetic return point memory (RPM) and complementary point memory (CPM) quantify how closely the domain pattern is reproduced after a full or half magnetization cycle, respectively. ${ }^{1}$ While magnetometry shows reproducible hysteresis loops, it is not obvious that such is true at the length scale of domains. Statistical characterization of how memory varies between microscopic and macroscopic length scales remains an important yet rarely studied aspect of material complexity. The occurrence of complete or partial microscopic memory is very important for achieving high performance domain wall logic and memory devices. ${ }^{2}$ RPM is apparently related to Barkhausen cascades, ${ }^{1}$ the magnitudes of which often exhibit scaling behavior. ${ }^{3}$ A compelling question is whether magnetic memory follows a functional behavior with length scale.

In this paper we present a technique for measuring length-scale-dependent magnetic memory in a thin $\mathrm{Co} / \mathrm{Pd}$ multilayer that exhibits perpendicular anisotropy. We do this by extending recent soft x-ray magnetic speckle metrology techniques and analyzing results in a scattering wave vector dependent fashion using angular cross correlation functions. We show that the memory has a maximum value near the initial reversal field of $H_{\mathrm{a}}=64 \mathrm{mT}$ and scattering wave vector $\mathbf{q}=0.0384 \mathrm{~nm}^{-1}$ where bubble domains have formed. At smaller and larger $\mathbf{q}$ corresponding to larger and smaller length scales respectively, and further from initial reversal, the memory decreases.

Previous q-integrated studies have correlated domain memory with roughness, ${ }^{1}$ an exchange-biased imprinted pattern, ${ }^{4}$ and nanostructuring. ${ }^{5}$ Magnetic memory is generally found to be large at initial reversal and decreases as the hysteresis loop is traversed, indicating that the domain configuration is similar at nucleation, but decreases as magnetization reversal progresses indicating that the domains evolve differently in each loop. ${ }^{6}$ Angulardependent, scattering vector resolved radial cross correlation analyses have recently been used to discover hidden symmetries in disordered colloidal glasses ${ }^{7}$ and to probe exchangebiased magnetic systems. ${ }^{8}$ Our results show statistically averaged memory at a length scale associated with bubble domains. 


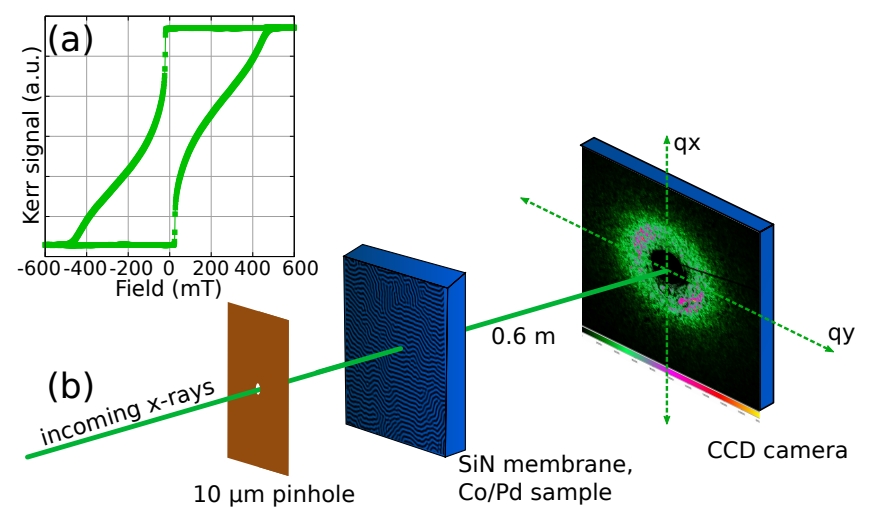

FIG. 1. (Color online). The polar Kerr signal is measured versus applied field in (a). In (b), the geometry for the coherent scattering experiment is shown.

Our sample was a $\mathrm{Co} / \mathrm{Pd}$ multilayer with a structure of $\mathrm{Ta}(2 \mathrm{~nm}) / \mathrm{Pd}(3 \mathrm{~nm}) /[\mathrm{Co}(0.5$ $\mathrm{nm}) / \mathrm{Pd}(0.7 \mathrm{~nm})]_{50} / \mathrm{Ta}(2 \mathrm{~nm})$ prepared by sputtering on a silicon nitride membrane 200 $\mathrm{nm}$ thick and $0.5 \mathrm{~mm}$ square. Sputtering was performed at room temperature with a pressure of 8 mTorr, conditions which lead to a moderate level of disorder. ${ }^{6}$ Sandwiching ultrathin Co layers between non-magnetic Pt or Pd layers leads to strong perpendicular anisotropy and out-of-plane magnetization. Figure 1 (a) shows the magnetization loop measured with polar magneto-optical Kerr effect. The rapid decrease of the magnetization near zero applied field followed by a monotonic change of the magnetization until saturation is evidence of abrupt domain nucleation followed by slower magnetization reversal. Transmission x-ray microscopy studies of similar CoPt multilayer films have revealed that the mechanism for magnetic reversal is bubble domain nucleation followed by growth of 'worm' domains into a full labyrinthine pattern of random orientation. ${ }^{9}$

The coherent soft x-ray scattering experiments were conducted on beamline 12.0.2.2 at the Advanced Light Source located at Lawrence Berkeley National Laboratory. Figure 1 (b) illustrates the geometry for the experiment, which was conducted at room temperature. The linearly polarized incident soft x-rays were tuned to the $\mathrm{Co}_{3}$ edge to achieve high scattering contrast to magnetic domain morphology. Coherence in the beam is established with a $10 \mu \mathrm{m}$ diameter pinhole approximately $7 \mathrm{~mm}$ upstream of the sample. The scattered light is collected in the forward direction with a charge coupled device (CCD) located $0.63 \mathrm{~m}$ downstream. The unscattered direct beam is blocked at the CCD to allow longer sampling of the diffuse light. To isolate the magnetic signal we subtract off an image taken 
at $\mathrm{H}= \pm 512 \mathrm{mT}$ that saturates the magnetization and thus contains only charge scattering signal. Figure 2 (a) shows the charge-subtracted scattering pattern collected at an applied field of $H_{\mathrm{a}}=256 \mathrm{mT}$. The annular scattering pattern arises from the random orientation of the labyrinth domains. ${ }^{6}$

To collect data we take $0.2 \mathrm{~s}$ images at each field in the sequence $\left(-512 \mathrm{mT}, \mathrm{H}_{\mathrm{a}}, \mathrm{H}_{\mathrm{a}}\right.$, $512 \mathrm{mT},-\mathrm{H}_{\mathrm{a}},-\mathrm{H}_{\mathrm{a}}$ ), repeated four times. Images collected at applied fields $\mathrm{H}_{\mathrm{a}}$ and $-\mathrm{H}_{\mathrm{a}}$ constitute a CPM measurement, whereas images collected at $\mathrm{H}_{\mathrm{a}}$ or $-\mathrm{H}_{\mathrm{a}}$ in different field cycles constitute an RPM measurement. The two consecutive images collected at $\mathrm{H}_{\mathrm{a}}$ or $-\mathrm{H}_{\mathrm{a}}$ are perfectly correlated except for uncorrelated read-out noise since the domains are static at room temperature and constant field. Collecting these images allows us to form a "quasi-autocorrelation function" (QACF) that consists of the cross correlation between two such images without an intervening field cycle. The QACF provides a much more robust normalization for our memory measurements than a true ACF since the read-out noise, which dominates the ACF when the scattering intensity is low, is uncorrelated.

In a speckle metrology measurement of domain memory, the reduced mutual coherence due to the change in the speckle pattern between image pairs taken at fields separated by a half- (CPM) and full-cycles (RPM) is calculated. In previous measurements of memory, ${ }^{1,4,6}$ mutual coherence is measured using two-dimensional, cross correlations in rectangular regions using periodic boundary conditions.

In this work we have developed a methodology whereby the angular symmetry in $\mathbf{q}$ allows for one-dimensional cross correlations to be formed as a function of azimuthal angle $\theta$ at fixed in-plane scattering vector $\mathbf{q}$. We define an angular cross correlation operation $C F(\mathbf{q}, \theta)$ between images $I_{1}$ and $I_{2}$ at scattering vector $\mathbf{q}$ by:

$$
C F(\mathbf{q}, \theta)=\sum_{\alpha} I_{1}(\mathbf{q}, \alpha) I_{2}(\mathbf{q}, \alpha+\theta) .
$$

where $\alpha$ is a dummy variable for summation. A QACF is formed if $I_{1}$ and $I_{2}$ are different images taken on the same field cycle and a CCF is formed when $I_{1}$ and $I_{2}$ are taken on different field cycles. The CCF and QACF were calculated in annular regions of $15 \mathrm{CCD}$ camera pixels wide $\left(\Delta \mathbf{q}=0.00131 \mathrm{~nm}^{-1}\right)$. The reduction of coordinates from the twodimensional image cross correlation to a one-dimensional angular cross correlation leads to line plots of cross correlations as shown in Fig. 2 (b). These correlation functions have an uncorrelated background with a zero-displacement peak centered at $\theta=0$ due to correlated 

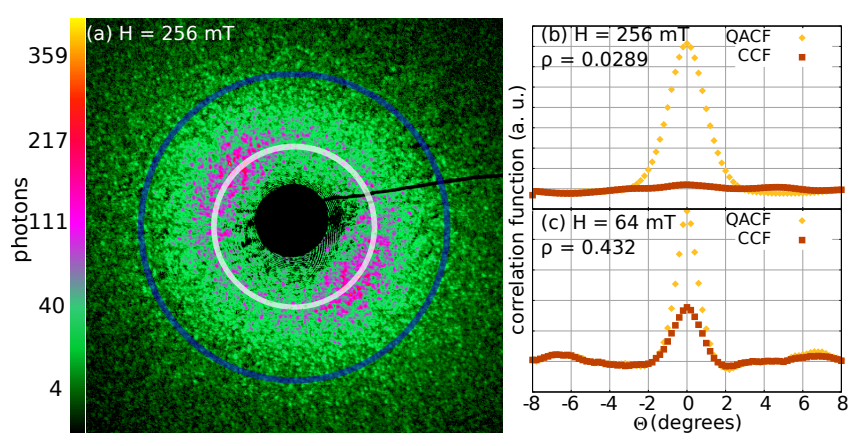

FIG. 2. (Color online). (a) The scattering pattern collected at $H_{\mathrm{a}}=256 \mathrm{mT}$. The color bar units are photons on a square root scale to emphasize the regions with fewer photons. Two 15 pixel wide annuli are shown at $\mathbf{q}=0.0202 \mathrm{~nm}^{-1}$ (light annulus) and $\mathbf{q}=0.0384 \mathrm{~nm}^{-1}$ (dark annulus). The spline-normalized QACF and CCF are calculated for the inner and outer annuli at $H_{\mathrm{a}}=256 \mathrm{mT}$ and $H_{\mathrm{a}}=64 \mathrm{mT}$ are shown in (b) and (c) respectively. The calculated memory using Eq. (2) is $\rho=0.0289$ in (b) $\rho=0.432$ in (c).

speckle. To isolate the zero-displacement peak we divide from the cross correlation functions a spline fit whose control points are chosen to exclude the peak. Fig. 2 (b)-(c) show splinenormalized CPM correlation functions collected at (b) $H_{\mathrm{a}}=256 \mathrm{mT}$ and $\mathbf{q}=0.0202 \mathrm{~nm}^{-1}$ (inner annulus in (b)) and (c) $H_{\mathrm{a}}=64 \mathrm{mT}$ and $\mathbf{q}=0.0384 \mathrm{~nm}^{-1}$ (outer annulus). The comparison of peak height at $\theta=0$ of Fig. 2 (c) illustrates the reduction of contrast of the $\mathrm{CCF}$ (squares) compared to the QACF (diamonds). The ratio of the CCF to the QACF is a visual representation of the magnetic memory.

To quantify the magnetic memory, we define a microscopic radial memory coefficient $\rho(\mathbf{q})$ between memory image pairs at scattering vector $\mathbf{q}$ as

$$
\rho(\mathbf{q})=\frac{\sum_{\theta} C C F(\mathbf{q}, \theta)}{\sum_{\theta} Q A C F(\mathbf{q}, \theta)},
$$

where $C C F(\mathbf{q}, \theta)$ and $Q A C F(\mathbf{q}, \theta)$ are the spline-normalized correlations defined in Eq. (1) and the summation is over the width of the peak of 15 pixels.

We find that averaging the cross correlation functions in Fig. 2 (b) of many CPM pairs before fitting with splines and then calculating a single value for the memory over the summed CPM pair decreases the noise compared to first calculating the memory for the pairs then averaging the memory values. Both methods show the same trends in the memory, so only the first method will be shown here. We also find that the results for the RPM 


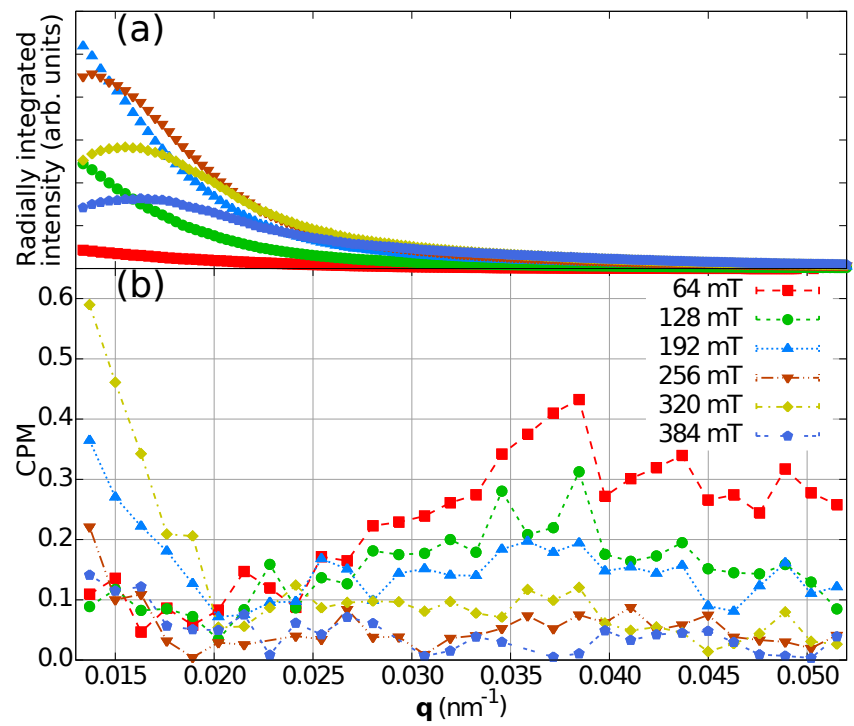

FIG. 3. (Color online). The (a) integrated scattering intensity and (b) CPM are plotted against scattering vector $\mathbf{q}$ for different applied fields.

measurement is similar in trends and behavior to the CPM measurement which we present here.

Figure 3 shows (a) the integrated intensity and (b) CPM versus scattering vector $\mathbf{q}$ for different applied fields between $64 \mathrm{mT}$ and $384 \mathrm{mT}$. The peak in the integrated scattering intensity begins at low $\mathbf{q}$ behind the beam block during initial reversal at low $\mathrm{H}_{\mathrm{a}}$, then increases with increasing field to larger $\mathbf{q}$ and peaks at $H_{\mathrm{a}}=384 \mathrm{mT}$ with $\mathbf{q}=0.0163 \mathrm{~nm}^{-1}$. From this we extract the length scale for an 'up'-'down' pair of domains to be $385 \mathrm{~nm}$ and a single domain to be $193 \mathrm{~nm}$. In the CPM vs $\mathbf{q}$ plot in (b), there is a maximum in the memory at small $\mathbf{q}\left(\mathbf{q}<0.020 \mathrm{~nm}^{-1}\right)$ for intermediate fields of $192-320 \mathrm{mT}$. This memory is an artifact of imperfect subtraction of saturation images leading to residual Airy fringes from the spatial filter pinhole and does not reflect high magnetic domain memory.

The memory plot at $64 \mathrm{mT}$ in Fig. 3 (b) (red squares) increases to a peak at $\mathbf{q}=0.0384$ $\mathrm{nm}^{-1}$. At this $\mathbf{q}$ as the applied field is increased, the peak decreases in magnitude to a background level at $H_{\mathrm{a}}=256 \mathrm{mT}$ indicating that, as the system further reverses, the memory at this length scale decreases. Although the scattering intensity at $\mathbf{q}=0.0384$ $\mathrm{nm}^{-1}$ is not large, the methodology outlined in this paper can extract information about the memory of magnetic domains at that length scale. ${ }^{10}$

With a peak in memory at a field near nucleation (64 $\mathrm{mT})$ and at a $\mathbf{q}$ approximately 
equal to the size of a single magnetic domain is suggestive that the peak is due to bubble domains. ${ }^{9}$ The bubble domains have high memory meaning that they are reproducibly the same size upon nucleation. As reversal proceeds the bubble domains evolve into labyrinths but, as seen in both imaging and scattering experiments, the evolution is random. ${ }^{6}$ The resulting random labyrinthine configurations exhibit low memory at all length scales. One may expect a peak in the memory at a $\mathbf{q}$ related to the average spacing between bubble nucleation points (approximately $700 \mathrm{~nm}),{ }^{9}$ but we are not able to access that scattering wave vector.

In conclusion, we present a method for extracting the q-dependent memory of a system. The technique can be used on any system that scatters in transmission with annuli of constant in-plane q. Using this method we have observed a peak in the memory as the system begins to reverse, which is a signature to bubble domain formation. This peak decreases as the magnetization is reversed indicating that the bubble domains form into labyrinth domains, which have no memory.

This work at LBNL was supported by the Director, Office of Science, Office of Basic Energy Sciences, of the U.S. Department of Energy under Contract No. DE-AC02-05CH11231. Work at UO was supported by NSF through Grant No. DMR-0506241. Work at UCSD was supported by DOE-BES Award No. DE-SC0003678.

\section{REFERENCES}

${ }^{1}$ M. S. Pierce, C. R. Buechler, L. B. Sorensen, J. J. Turner, S. D. Kevan, E. A. Jagla, J. M. Deutsch, T. Mai, O. Narayan, J. E. Davies, K. Liu, J. H. amd K. M. Chesnel, J. B. Kortright, O. Hellwig, and E. E. Fullerton, Phys. Rev. Lett., 94, 017202 (2005).

${ }^{2}$ M. Hayashi, L. Thomas, R. Moriya, C. Rettner, and S. S. P. Parkin, Science, 320, 209 (2008).

${ }^{3}$ G. Durin and S. Zapperi, Phys. Rev. Lett., 84, 4705 (2000).

${ }^{4}$ K. Chesnel, E. E. Fullerton, M. J. Carey, J. B. Kortright, and S. D. Kevan, Phys. Rev. B, 78, 132409 (2008).

${ }^{5}$ G. Beutier, A. Marty, F. Livet, A. Haznar, E. Dudzik, S. Stanescu, V. Chamard, and G. van der Laan, New J. Phys., 11, 113026 (2009).

${ }^{6}$ K. A. Seu, R. Su, S. Roy, D. Parks, E. Shipton, E. E. Fullerton, and S. D. Kevan, New J. 
Phys., 12, 035009 (2010).

${ }^{7}$ P. Wochner, C. Gutt, T. Autenrieth, T. Demmer, V. Bugaev, A. D. Ortiz, A. Duri, F. Zontone, G. Grübel, and H. Dosch, Proceedings of the National Academy of Sciences, 106, 11511 (2009).

${ }^{8}$ K. Chesnel, J. A. Nelson, S. D. Kevan, M. J. Carey, and E. E. Fullerton, Phys. Rev. B, 83, 054436 (2011).

${ }^{9}$ J. E. Davies, O. Hellwig, E. E. Fullerton, G. Denbeaux, J. B. Kortright, and K. Liu, Phys. Rev. B, 70, 224434 (2004).

${ }^{10}$ To check our analysis method, we have altered the annular width from 5-50 px $(\Delta \mathbf{q}=$ $0.000435-0.00435 \mathrm{~nm}^{-1}$ ) and the peak at $64 \mathrm{mT}$ and $\mathbf{q}=0.0384 \mathrm{~nm}^{-1}$ persists but larger annuli smooth out the fine q-resolved details. We have also correlated two saturation images that contain only charge scattering; they do not contain the peak at $\mathbf{q}=0.0384$ $\mathrm{nm}^{-1}$ which indicates that it is magnetic in origin. 


\section{DISCLAIMER}

This document was prepared as an account of work sponsored by the United States Government. While this document is believed to contain correct information, neither the United States Government nor any agency thereof, nor The Regents of the University of California, nor any of their employees, makes any warranty, express or implied, or assumes any legal responsibility for the accuracy, completeness, or usefulness of any information, apparatus, product, or process disclosed, or represents that its use would not infringe privately owned rights. Reference herein to any specific commercial product, process, or service by its trade name, trademark, manufacturer, or otherwise, does not necessarily constitute or imply its endorsement, recommendation, or favoring by the United States Government or any agency thereof, or The Regents of the University of California. The views and opinions of authors expressed herein do not necessarily state or reflect those of the United States Government or any agency thereof or The Regents of the University of California. 